\title{
Genetics of Penicillin Titre in Lines of Aspergillus nidulans Selected through Recurrent Mutagenesis
}

\author{
By I. N. SIMPSON† AND C. E. CATEN* \\ Department of Genetics, University of Birmingham, Birmingham B15 2TT
}

(Received 31 March 1980)

\begin{abstract}
Genetic analyses of mutations for increased penicillin titre were carried out on two strains of Aspergillus nidulans isolated after independent programmes of recurrent mutation and selection, similar to those used industrially for strain improvement. Both selected strains were stable in terms of colony morphology and penicillin titre. Backcrosses to the unselected ancestor indicated that the increased yield of each strain was due to an induced polygenic system with both additive and non-additive gene action. The titre-increasing mutations in one strain (A6-9) were recessive to their wild-type alleles, while those in the other (B6-27) were either semi-dominant or showed ambidirectional dominance. The diploid between A6-9 and B6-27 had a titre less than either component haploid, suggesting that the mutations in the two strains involved different genes. Strain B6-27 carried a translocation between chromosomes III and VII which had been induced during the selection programme. This investigation complements previous work on the genetics of penicillin production in $A$. nidulans and Penicillium chrysogenum and the results are directly relevant to industrial strain improvement by hybridization of divergent strains.
\end{abstract}

\section{INTRODUCTION}

Major increases in the yields of antibiotics have been achieved in industrial strain development programmes through the use of repeated cycles of mutation and selection (Alikhanian, 1962; Elander, 1967; Demain, 1973; Elander \& Espenshade, 1976). Little is known, however, about the genetic changes responsible for increased productivity (Hopwood \& Merrick, 1977) despite the fact that such information should facilitate further strain improvement by mutational or recombinational techniques. This deficiency may be attributed to the general success of mutation and selection procedures which has made major investment in studies of the genetics of the producing organisms commercially unnecessary. However, the increasing difficulty of obtaining superior cultures by empirical mutation breeding (Demain, 1973) and the advent of genetic engineering (Hopwood \& Merrick, 1977) stress the need for more information about the genetic determination of antibiotic production.

Following the demonstration of the parasexual cycle in Penicillium chrysogenum (Pontecorvo \& Sermonti, 1954), attempts were made to determine the genetic basis of the high penicillin titres of improved strains and to increase productivity further by breeding (Sermonti, 1959, 1961; Alikhanian \& Kameneva, 1961; Macdonald et al., 1963a, b, 1964, 1965; Elander et al., 1973). These investigations established the nuclear basis and generally recessive nature of titre-increasing mutations and indicated some allelism between the mutations in different high-yielding strains. Considerable difficulties were encountered, however, when segregants from diploids between independent high-yielding strains were examined. These segregants proved generally to be of one or the other parental phenotype with respect to both markers and titre and few conclusions could be drawn concerning the

$\dagger$ Present address: Microbiology Division, Glaxo Group Research Ltd, Greenford, Middlesex UB6 0HE. 
Table 1. Details of Aspergillus nidulans strains used

Strain no.

7-142 (A)

$7-151$ (B)

A6-9

B6-27

$7-21$

$7-141$

$7-145$

7-154
Genotype*

biA1

$w A 3 ;$ pabaA1

biAl; $\Sigma$ penA

wA3; pabaA1; penB

suAadE20, adE20, yA2; AcrA1; phenA2; pyro $A 4$; lysB5; sB3; nicB8; riboB2 sUAadE20, adE20, biA1; AcrA1; sA4; pyro $A 4$; pA2; lac $A 1$; nicB8; riboB2 suAadE20, adE20, yA2; wA3; galA1; pyro $A 4$; facA303; sB3; nicB8; riboB2 wild-type
Origin

See Simpson \& Caten (1979a); ATCC 42369

See Simpson \& Caten (1979a); ATCC 42370

See Simpson \& Caten (1979b)

See Simpson \& Caten $(1979 b)$

FGSC 68

FGSC 375

FGSC 288

Recombinant from 7-142 $\times$ 7-151

* $\Sigma$ pen $A$ and $\Sigma$ pen $B$ denote induced mutations for increased penicillin titre accumulated during selection programmes A and B, respectively (see Simpson \& Caten, 1979b). Other gene symbols are standard for A. nidulans (Clutterbuck, 1973).

number of titre-increasing mutations involved and their interaction (Macdonald et al., 1964, 1965). This phenomenon of parental genome segregation probably resulted from differences in chromosome structure produced by the repeated mutagenic treatments to which the parent strains had been subjected (Macdonald, 1968). Ball (1971, $1973 a$ ) was able to avoid this difficulty by careful choice of parental strains, but this limited his analysis to single-step titre mutants and prevented extrapolation to industrial strains.

Many strains of Aspergillus nidulans produce penicillin (Holt \& Macdonald, 1968a) and studies with this organism have added considerably to knowledge of the genetics of penicillin production. These studies involved either individual major mutations affecting titre or natural allelic differences (for review, see Macdonald \& Holt, 1976) and therefore were not directly relevant to the genetic basis of increased titre in industrial strains. For this purpose, strains selected through repeated mutagenesis in a manner comparable to that used industrially for strain improvement are required. Two such strains of $A$. nidulans, A6-9 and B6-27, have been derived through six cycles of mutagenesis and selection for increased penicillin production (Simpson \& Caten, 1979b). Comparison of A6-9 and B6-27 with their unselected ancestors in a replicated trial at the end of the selection programmes showed around a threefold increase in productivity in each selection line. This paper reports analyses of the genetic changes underlying the increased productivity of strains A6-9 and B6-27. These changes proved to have a polygenic basis necessitating the use of quantitative methods (Caten \& Jinks, 1976; Caten, 1979). Improved strains of $P$. chrysogenum are frequently somatically unstable (Backus \& Stauffer, 1955) and are thought to carry various chromosomal rearrangements (Muchnik, 1961; Macdonald, 1968). These two aspects of their phenotype are probably related since at least certain types of chromosomal aberration may produce instability (Roper, 1973). Because of the significance of instability in industrial fungi (Ball \& Azevedo, 1976), strains A6-9 and B6-27 were tested for clonal stability and the presence of translocations.

\section{METHODS}

Strains. Eight strains of $A$. nidulans were used (Table 1). These were all descended from the 'Glasgow' wild-type, NRRL 194 (Barratt et al., 1965). The origins of the ancestral strains 7-142 (biAl) and 7-151 (pabaA1; wA3) and their high-titre derivatives A6-9 (biA1) and B6-27 (pabaA1; wA3) have been described previously (Simpson \& Caten, 1979 $a, b$ ). For ease of presentation, strains 7-142 and 7-151 will be referred to as A and B, respectively. Strains 7-21, 7-141 and 7-145 are master strains which carry at least one marker on each chromosome (McCully \& Forbes, 1965). Strain 7-154 is a wild-type recombinant derived from the cross $\mathrm{A} \times \mathrm{B}$. The penicillin titre-increasing mutations (' + ' mutations) accumulated in A6-9 and B6-27 are 
Table 2. Summary of analyses of variation in penicillin titre $\left(\log u \mathrm{ml}^{-1}\right)$ among populations of single conidiospore isolates from strains A, B, A6-9 and B6-27

(a) Tests of significance

\begin{tabular}{|c|c|c|c|c|c|c|}
\hline \multirow[b]{2}{*}{ Strain } & \multicolumn{2}{|c|}{ Between isolates } & \multicolumn{2}{|c|}{ Between blocks } & \multicolumn{2}{|c|}{ Isolates $\times$ blocks } \\
\hline & d.f. & M.S. $\neq$ & d.f. & M.S. & d.f. & M.S. \\
\hline $\mathrm{A} \dagger$ & 48 & 0.015 & 1 & $0 \cdot 000$ & 48 & 0.019 \\
\hline A6-9 & 47 & 0.003 & 1 & $0 \cdot 062^{*}$ & 47 & 0.002 \\
\hline $\mathrm{B} \dagger$ & 51 & 0.002 & 1 & $0.032 *$ & 51 & 0.002 \\
\hline B6-27 & 47 & 0.001 & i & $0.022^{*}$ & 47 & 0.001 \\
\hline
\end{tabular}

(b) Components of variance§

$\begin{array}{lccc}\text { Strain } & \sigma_{G^{2}} & \sigma_{B^{2}} & \sigma_{E^{2}} \\ \mathrm{~A} \dagger & 0.000 & 0.000 & 0.019 \\ \mathrm{~A} 6-9 & 0.001 & 0.000 & 0.002 \\ \mathrm{~B} \dagger & 0.000 & 0.000 & 0.002 \\ \mathrm{~B} 6-27 & 0.000 & 0.000 & 0.001\end{array}$

d.f., degrees of freedom; M.S., mean square.

$* P<0.001$.

$\dagger$ Data from Simpson \& Caten (1979a).

$\mp$ Tested against isolates $\times$ blocks M.S.

$\S \sigma_{G}{ }^{2}, \sigma_{B}{ }^{2}$ and $\sigma_{E}{ }^{2}$ are estimates of the variance due to genetic, experimental block design and environmental efiects, respectively.

denoted $\Sigma$ pen $A$ and $\Sigma$ pen $B$, respectively. With this exception, the gene symbols are standard for $A$. nidulans (Clutterbuck, 1973).

General. Media, methods of culture, fermentation and bioassay procedures and statistical analyses were all as described previously (Simpson \& Caten, 1979a).

Genetical methods. All sexual crosses were carried out by single perithecium analysis (Pontecorvo et al., 1953) following the procedure of Merrick \& Caten (1975). Diploids were isolated from balanced heterokaryons by Roper's (1952) method. For haploidization, diploid strains were point-inoculated on to Czapek agar containing complete medium supplement (Simpson \& Caten, 1979a) and, as a haploidizing agent, either $p$-fluorophenylalanine at a final concentration of $80 \mu \mathrm{g} \mathrm{ml}^{-1}$ (Lhoas, 1961) or Du Pont benlate (Pan Britannica Industries Ltd, Britannica House, Waltham Cross, Herts.) at a final concentration of $1.2 \mu \mathrm{g} \mathrm{ml}^{-1}$ (Hastie, 1970). Segregants were recognized by spore colour and not more than one sector of each colour was isolated from each diploid colony. Procedures for the determination of segregant genotypes were standard for A. nidulans (Pontecorvo et al., 1953; Clutterbuck, 1974).

Testing for chromosomal rearrangements. Tests for the presence of translocations in the ancestral and selected strains were made using two criteria: (i) the frequency of colonies with abnormal morphology in the progeny of sexual crosses (Upshall \& Käfer, 1974) and (ii) reassortment of markers during haploidization of heterozygous diploids (Käfer, 1962). Strains A, B, 7-21, 7-141 and 7-145 (Table 1) all possess the standard chromosome complement and were used as reference strains in these tests. The following sexual crosses were made: A $\times$ B, A6-9 $\times$ B, B6-27 $\times$ A, A6-9 $\times$ B6-27. Ascospores were plated at low density from these crosses and the frequencies of small, abnormal colonies in the progenies were determined. For the haploidization analysis, a heterozygous diploid was constructed between each of the ancestral or selected strains and an appropriate master strain as follows: A/7-145, B/7-141, A6-9/7-21, B6-27/7-21.

\section{RESULTS}

\section{Stability of strains A6-9 and B6-27}

Conidial platings of strains A, B, A6-9 and B6-27 were examined for segregation of different colony types comparable to that shown by improved strains of $P$. chrysogenum (Backus \& Stauffer, 1955). No segregation was observed with any of the strains. To test the stability of A6-9 and B6-27 with respect to penicillin titre, 50 single conidial isolates from each strain were each fermented in two replicate flasks. The conidial populations from the two strains were fermented in separate experiments and five replicate flasks of the appropriate ancestor (A or B) were included. Analyses of variance for the data from these two 


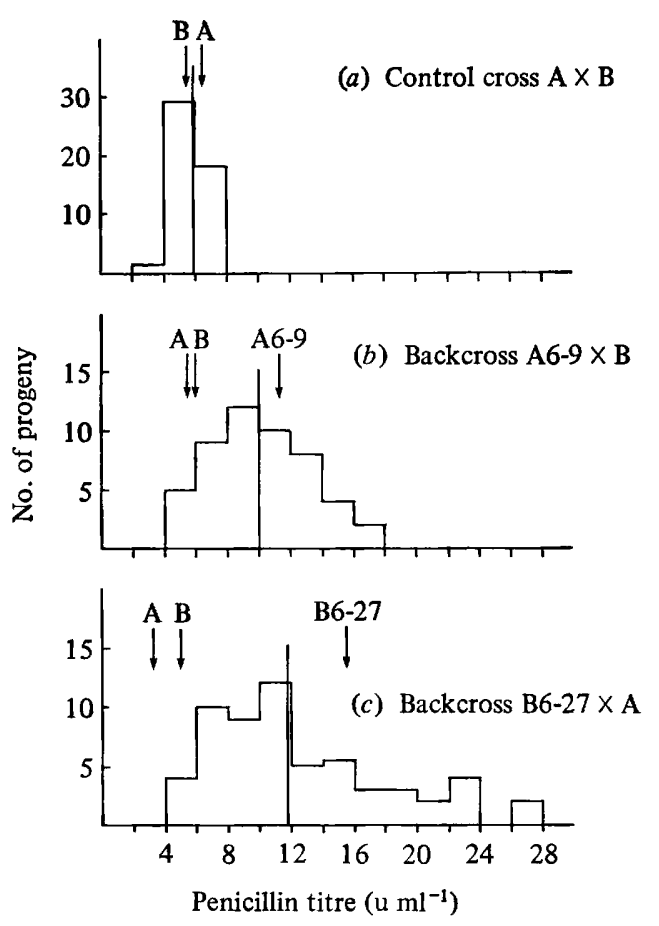

Fig. 1. Distribution of penicillin titres among progeny from crosses $\mathrm{A} \times \mathrm{B}(a), \mathrm{A} 6-9 \times \mathrm{B}(b)$ and B6-27 $\times \mathrm{A}(\mathrm{c})$. The titres of $\mathrm{A}$ and $\mathrm{B}$ and the appropriate selected parent are indicated by arrows. The bars indicate the progeny mean titres.

fermentation experiments indicated no differences in titre among sister clones of A6-9 or B6-27 (Table 2). Data previously obtained for conidial populations from strains A and B (experiments 5 and 1, respectively, in Simpson \& Caten, 1979a) are included in Table 2 for comparison. Clearly, the selected strains are no more unstable, as reflected by variation in penicillin titre among sister clones, than their ancestors.

The mean titres of the conidial populations from the selected strains (A6-9 $=12.0$ $\mathrm{u} \mathrm{ml} \mathrm{m}^{-1}, \mathrm{~B} 6-27=11.0 \mathrm{u} \mathrm{ml} \mathrm{m}^{-1}$ ) were significantly higher than those of the ancestral strains fermented in the same experiment $\left(A=5.8 \mathrm{u} \mathrm{ml}^{-1}, \mathrm{~B}=6.4 \mathrm{u} \mathrm{ml}^{-1}\right)$, further confirming the response achieved in the two selection programmes.

\section{Backcrosses of improved strains to the ancestor}

To determine the general nature of the induced genetic changes the two improved strains were each crossed back to the ancestor. Because of the need to retain heterozygosity of the markers in order to recognize hybrid perithecia it was not possible to make the true backcrosses, A6-9 $\times \mathrm{A}$ and B6-27 $\times \mathrm{B}$. However, the crosses A6-9 $\times \mathrm{B}$ and B6-27 $\times \mathrm{A}$ could be made and, since A and B had been initially made isogenic (Simpson \& Caten, 1979a), these should be equivalent to the backcrosses. As a control on their isogenicity, strains A and $B$ were also crossed. Around 50 single ascospore progeny were selected at random from A6-9 $\times \mathrm{B}$ and from B6-27 $\times \mathrm{A}$ and their genotypes were determined with respect to the markers $b i A, p a b a A$ and $w A$. For $\mathrm{A} \times \mathrm{B}$, six progeny were selected for each of the eight marker genotypes. The markers segregated and recombined as expected in all three crosses. Each of the three progeny populations was assayed in a separate experiment with the progeny and parental strains (A, B, A6-9 and B6-27) each fermented in two replicate flasks arranged as two randomized blocks.

The distributions of progeny titres in the control and two backcross populations are 
Table 3. Summary of analyses of variation in penicillin titre $\left(\log u \mathrm{ml}^{-1}\right)$ among progeny populations from the backcrosses $\mathrm{A} 6-9 \times \mathrm{B}$ and $\mathrm{B} 6-27 \times \mathrm{A}$ and from the control cross $\mathrm{A} \times \mathrm{B}$

(a) Tests of significance

\begin{tabular}{|c|c|c|c|c|c|c|c|c|c|c|}
\hline \multirow[b]{2}{*}{ Cross } & \multicolumn{2}{|c|}{ Between progeny } & \multicolumn{2}{|c|}{$\begin{array}{l}\text { Between marker } \\
\text { genotypes }\end{array}$} & \multicolumn{2}{|c|}{$\begin{array}{c}\text { Within marker } \\
\text { genotypes }\end{array}$} & \multicolumn{2}{|c|}{ Between blocks } & \multicolumn{2}{|c|}{ Progeny $\times$ blocks } \\
\hline & d.f. & M.S. & d.f. & M.S. & d.f. & M.S. & d.f. & M.S. & d.f. & M.S. \\
\hline $\mathbf{A} \times \mathbf{B}$ & 47 & 0.010 & 7 & $0 \cdot 016$ & 40 & 0.009 & 1 & $0.149 *$ & 47 & 0.012 \\
\hline A6-9 $\times$ B & 48 & $0.038^{*}$ & 5 & 0.051 & 43 & $0.036^{*}$ & 1 & $0.035^{*}$ & 48 & 0.007 \\
\hline B6-27 $\times$ A & 52 & $0.070^{*}$ & 6 & 0.056 & 46 & $0.072^{*}$ & 1 & 0.019 & 52 & 0.013 \\
\hline
\end{tabular}

(b) Components of variance $\dagger$

$\begin{array}{lllc}\text { Cross } & \sigma_{G}^{2} & \sigma_{B}^{2} & \sigma_{E^{2}} \\ \mathrm{~A} \times \mathrm{B} & 0.000 & 0.003 \ddagger & 0.012 \\ \mathrm{~A} 6-9 \times \mathrm{B} & 0.016 \ddagger & 0.001 \ddagger & 0.007 \\ \mathrm{~B} 6-27 \times \mathrm{A} & 0.028 \ddagger & 0.000 & 0.013\end{array}$

d.f., degrees of freedom; M.S., mean square.

$* P<0.001$.

$\dagger \sigma_{G}{ }^{2}, \sigma_{B}{ }^{2}$ and $\sigma_{E}{ }^{2}$ are estimates of the variance due to induced genetic, experimental block design and environmental effects, respectively.

$\ddagger$ Significantly different from zero.

shown in Fig. 1. All three distributions were continuous with a single mode, and there was no indication of the segregation of a major gene affecting penicillin titre in any cross. While the distributions for $A \times B$ and A6-9 $\times B$ were approximately normal, the distribution for B6-27 $\times$ A was positively skewed. The mean titres of the two backcross populations were intermediate between their parents and higher than the mean of the control population or the titres of the ancestors raised in the same experiment, demonstrating that the induced increases in titre were transmitted to the next sexual generation and hence were genetically determined. The range of progeny titres in each backcross was markedly greater than in the control cross (Fig. 1), the latter being similar to that observed in the conidial populations. This variability of the backcross populations may be attributed to the simultaneous segregation of several titre-increasing mutations induced in A6-9 and B6-27 by the repeated mutagenic treatment.

Analyses of variance for the three populations confirmed this interpretation (Table 3). The variation among the progeny from each cross was partitioned into that between and that within marker genotypes. In no case was the between marker genotypes mean square significant when tested against the within marker genotypes mean square (Table $3 a$ ), indicating that the markers $b i A, p a b a A$ and $w A$ had no effect on penicillin titre. The within marker genotypes item, however, was significantly greater than the replicate error variance (progeny $\times$ blocks mean square) for both backcrosses but not for the control cross (Table $3 a$ ). Since there was no effect of the markers, the within and between marker genotypes mean squares could be combined to give a between progeny mean square with the expectation $\sigma_{E}{ }^{2}+2{\sigma_{G}}^{2}$, where $\sigma_{E}{ }^{2}$ is the environmental variance and $\sigma_{G}{ }^{2}$ is the induced genetic variance. Estimates of $\sigma_{G}{ }^{2}$ (Table $3 b$ ) confirmed that induced mutations were the major source of the observed phenotypic variation in both backcrosses (Fig. 1 $b, c$ ) but, as expected, were not present in the control cross (Fig. 1a). The environmental component $\left(\sigma_{E}{ }^{2}\right)$ was homogeneous over the three populations (Table $3 b$ ) and this again indicates that increased titre is not associated with increased instability. These analyses therefore confirm the segregation of alleles affecting penicillin titre in both backcrosses and indicate that these genetic differences must have been induced during the selection programmes since the ancestors $\mathrm{A}$ and $\mathrm{B}$ do not differ for_alleles affecting this character. 
Table 4. Dominance and allelism of induced mutations for increased penicillin titre in $\mathrm{A} 6-9$ and $\mathrm{B} 6-27$

(a) Mean titres of haploid and diploid strains

Strain

$7-154$

A

B

A6-9

B6-27

A/B

A6-9/B

B6-27/A

A6-9/B6-27

Item

(i) Dominance of $\Sigma$ penA

(ii) Dominance of $\Sigma$ pen $B$

(iii) Allelism of $\Sigma$ pen $A$ and $\Sigma p e n B$

Ns, Not significant.

* Students $t$ test.

$\dagger I$ is the mean titre of the unselected strains 7-154, A, B and A/B.

$\ddagger T$ is the predicted titre of A6-9/B6-27. See section on Dominance and allelism of the induced mutations for details.

Tests for non-additive gene effects were applied to the data for the two backcrosses as described by Merrick \& Caten (1975) and Caten (1979). In both crosses the progeny mean was significantly higher than the mid-parental value $(\mathrm{A} 6-9 \times \mathrm{B}$ : $[i]=-0.064, P=0.01-$ $0.001 ; \mathrm{B} 6-27 \times \mathrm{A}:[i]=-0.170, P=<0.001)$ indicating non-additive gene action. The excess of high-titre progeny suggests that the individual effects of at least some of the mutations are enhanced when they are separated into low-titre backgrounds, i.e. in combination in the selected strains the ' + ' mutations express a degree of duplicate epistasis

\section{Dominance and allelism of the induced mutations}

The dominance of the ' + ' mutations in A6-9 and B6-27 and the extent of allelism between the mutations in the two strains were investigated in a single fermentation experiment. For these purposes, four diploids - A/B, A6-9/B, B6-27/A and A6-9/B6-27 - were constructed and their titres were determined along with those of the haploids. A, B, A6-9, B6-27 and 7-154. These nine strains were each fermented in ten replicate flasks arranged in two randomized blocks.

The mean titres of the related, unselected haploids A, B and 7-154 were homogeneous, again showing that the markers have no effect on penicillin production (Table 4). Diploid A/B did not differ significantly from these haploid strains. This indicates that ploidy per se has no effect, in agreement with the finding in P. chrysogenum (Sermonti, 1959). Accordingly, the results for these four strains were pooled to give an estimate $(I)$ of the average perform- 
ance of the unselected genome $\left(I=7 \cdot 4 \mathrm{u} \mathrm{ml}^{-1}\right)$. Since ploidy per se does not influence titre, the average dominance of the induced mutations could be determined by comparing the diploids between the improved and the ancestral haploids with their component haploids. [The observed titre of A6-9 in this experiment $\left(11.7 \mathrm{u} \mathrm{ml}^{-1}\right)$ was unexpectedly low; nevertheless this value has been used for the subsequent comparisons.] The significance of these comparisons was determined by $t$ tests. Diploid A6-9/B gave a titre which was significantly less than both that of A6-9 and the mean of its component haploids but which did not differ from the titre of the unselected genome $I$ [Table $4 b(i)]$. The '+' mutations in A6-9 are therefore recessive to their wild-type alleles. Diploid B6-27/A behaved differently, giving an intermediate titre which was significantly different from both B6-27 and $I$ but not from the mean of its component haploids [Table $4 b(i i)$ ]. Either all the induced changes in B6-27 are semi-dominant or some ' + ' mutations are dominant and others recessive to their wild-type alleles.

In assessing the extent of allelism of the '+' mutations in A6-9 and B6-27 the dominance of these mutations must be considered. We may use the dominance relationships observed in diploids A6-9/B and B6-27/A to predict the titre of diploid A6-9/B6-27 on the assumptions that the mutations in the two improved strains are not allelic and that they do not interact. This predicted titre $(T)$ is given by:

$$
T=I+[(\mathrm{A} 6-9 / \mathrm{B})-I]+[(\mathrm{B} 6-27 / \mathrm{A})-I]=11 \cdot 0 \mathrm{u} \mathrm{ml}^{-1}
$$

$T$ sets a lower limit on the titre and any allelism would be expected to raise it above this limit. With complete allelism, A6-9/B6-27 should have a titre equivalent to that of the selected haploids. However, since A6-9 and B6-27 differ significantly from each other [Table $4 b(i i i)$ ] and have different dominance properties, their mutations are unlikely to be completely allelic. The titre of A6-9/B6-27 was less than that of B6-27 but was not significantly different from the predicted titre $T$ [Table $4 b(i i i)$. (The lack of any difference between A6-9/B6-27 and A6-9 probably results from the anomalously low titre of A6-9 in this experiment.) The agreement between the observed and predicted titres for A6-9/ B6-27 indicates that the '+' mutations induced in the two improved strains are not allelic. Interaction between the mutations is suggested by the fact that the observed titre is somewhat less than the predicted.

\section{Chromosomal organization}

Small abnormal colonies occurred amongst the progeny of the sexual cross $\mathbf{A} \times \mathbf{B}$ at a frequency less than $0.25 \%$. This is consistent with their frequency in known translocationfree crosses (Upshall \& Käfer, 1974) and confirms that A and B both carry the standard chromosome complement. Cross A6-9 $\times \mathrm{B}$ also gave this low frequency of abnormal progeny, indicating that A6-9 has retained the standard chromosomal organization. However, in both crosses involving B6-27 (B6-27 $\times$ A and A6-9 $\times$ B6-27) around $4 \%$ of the progeny were abnormal. This frequency is characteristic of crosses heterozygous for a translocation (Upshall \& Käfer, 1974) and indicates the introduction of a translocation during mutation programme B (Simpson \& Caten, 1979b).

Around 100 segregants from each of diploids A/7-145, B/7-141 and A6-9/7-21 and 187 segregants from diploid B6-27/7-21 were characterized in the haploidization analyses. All unlinked markers reassorted in the haploids from the first three diploids, confirming that strains A, B and A6-9 were translocation free. With B6-27/7-21, however, the markers phen $A$ (chromosome III) and nicB (chromosome VII) failed to reassort. All other markers behaved as expected. This confirmed the presence of a translocation in B6-27 and identified that translocation as involving chromosomes III and VII. 


\section{DISCUSSION}

The present study was designed to investigate the genetics of penicillin production in strains of $\boldsymbol{A}$. nidulans isolated after recurrent mutation and selection procedures comparable to those used industrially for strain improvement. In this way it was hoped that the results would be directly relevant to the genetics of antibiotic production in industrial strains, particularly penicillin-producing strains of $P$. chrysogenum. The genetics of penicillin production has previously been examined in $P$. chrysogenum and $A$. nidulans. The majority of studies with $P$. chrysogenum (Sermonti, 1959, 1961; Alikhanian \& Kameneva, 1961; Macdonald et al., 1963a, b, 1964, 1965; Elander, 1967; Elander et al., 1973) have employed highly selected strains and hence were of direct industrial relevance. Unfortunately, the results obtained and the conclusions that could be drawn were limited by lack of knowledge about the basic genetics of the organism and by technical difficulties of instability and parental genome segregation. These technical difficulties can be overcome by the use of closely related parental strains and by modified technical procedures (Ball, 1971, 1973a), but the former requirement precludes the analysis of divergent industrial strains. In contrast, the work with $A$. nidulans has not been hampered by technical difficulties and has yielded clear results. However, it did not aim to provide information on the genetics of highly selected strains but concentrated on single major mutations affecting penicillin titre either positively (Ditchburn et al., 1976) or negatively (Holt et al., 1976) and on natural variation in penicillin production (Holt \& Macdonald, 1968b; Merrick \& Caten, 1975; Merrick, $1975 a, b)$. The present results will be discussed in relation to these previous investigations of the genetics of penicillin production.

The continuous, unimodal nature of the progeny distributions in both backcrosses (Fig. 1) indicates that the titre increases in A6-9 and B6-27 are each determined by several genes which recombine at meiosis to produce progeny with intermediate phenotypes. It has been generally assumed from the multistep origin of industrial strains of $P$. chrysogenum that polygenic systems are responsible for their increased potency (Sermonti, 1969; Roper, 1973; Elander \& Espenshade, 1976); parental genome segregation, however, has prevented detailed analyses of these systems in crosses. Since in small populations apparently continuous phenotypic distributions can be produced by the segregation of a few genes (Thoday \& Thompson, 1976), little can be deduced from the backcrosses about the number of mutations involved in each selected strain, other than it must be more than one or two. However, the selection responses suggested a minimum of three and six mutational steps in the development of A6-9 and B6-27, respectively (Simpson \& Caten, 1979b). The polygenic system produced in each selected strain by these mutations shows both additive and non-additive gene action, although the latter effects are small. None of these ' + ' mutations appears to be linked to the markers $b i A$, pabaA or $w A$ as there were no differences between the marker genotypes in either backcross (Caten \& Jinks, 1976).

The ' + ' mutations in A6-9 appear to be recessive, while those in B6-27 appear to be semi-dominant. Since each strain carries several mutations, the tests show only average dominance and the observed semi-dominance may reflect a net balance between recessive and dominant ' + ' mutations. Comparable tests with $P$. chrysogenum have shown that ' + ' mutations are generally recessive (Sermonti, 1959; Macdonald et al., 1963b). However, semi-dominance has been reported for the penC3 mutation in $A$. nidulans (Ditchburn et al., 1976).

Consideration of the large number of loci that are potentially capable of affecting penicillin titre (Simpson \& Caten, 1979a) suggests that independently derived improved strains should largely carry mutations in different genes. This expectation is supported by the low titre of diploid A6-9/B6-27, which indicated that the mutations in the two improved strains are not allelic. Comparable studies of heterozygous diploids between independent high-yielding strains of $P$. chrysogenum gave conflicting results. Some diploids had titres 
less than either parent indicating non-allelism (Sermonti, 1959), others had titres comparable to the lower yielding parent suggesting extensive allelism (Macdonald et al., 1964), while in other cases independently isolated diploids between the same immediate parents gave a range of titres making it difficult to draw any conclusions about allelism (Macdonald et al., 1965). Three major mutations raising penicillin yield in A. nidulans - penA1, penB2 and penC3 - all involved different genes (Macdonald \& Holt, 1976) and the variation in penicillin production among natural isolates of this fungus was determined by many genes (Merrick, 1975b). Overall, therefore, these genetic analyses confirm that '+' mutations can occur at many loci and that different genes are likely to be responsible for the increased productivity of independently selected lines.

Haploidization analysis of diploids between closely related strains of $P$. chrysogenum indicated that many pairs of markers assort independently and established several linkage groups (Sermonti, 1957; Ball, 1971). In contrast, where divergent industrial strains were involved there was a strong tendency for the markers to retain their parental combinations (Macdonald et al., 1964). This different behaviour is believed to result from chromosomal rearrangements in the divergent strains which prevent reassociation at haploidization between the linkage groups concerned (Macdonald, 1968; Ball, 1971). Since the divergent strains were all derived from the same ancestor (Macdonald et al., 1964), the chromosomal rearrangements must have been induced during the mutation and selection programmes. The presence of at least certain types of chromosomal rearrangements in industrial strains of $P$. chrysogenum is further indicated by their mitotic instability, which may be traced back to Q176 in the Wisconsin family (Backus \& Stauffer, 1955; Ball, 1973b). Partial chromosomal duplications are a major cause of similar instabilities in $A$. nidulans (Roper, 1973). Whether these various chromosomal rearrangements were induced and isolated coincidently with titre-increasing mutations or whether they themselves contribute to the increased productivity has not been established.

The mutagens used to derive A6-9 and B6-27 were chosen to minimize the risk of gross chromosomal change (Simpson \& Caten, 1979a). The present results indicate that this aim was largely realized. Strains A6-9 and B6-27 were as stable as their ancestors, suggesting that they did not carry any major duplications; A6-9 was also free of translocations, but a translocation between chromosomes III and VII had been induced in B6-27. The sample size is too small to determine whether the induction of a translocation in mutation programme B and not in programme A reflects a difference between the two mutagens used ethyl methanesulphonate and near-u.v. in the presence of 8-methoxypsoralen, respectively (Simpson \& Caten, 1979a, b). Since neither of the sexual crosses heterozygous for the translocation (B6-27 $\times \mathrm{A}$ and A6-9 $\times$ B6-27) produced progeny with the characteristic 'crinkled' morphology of strains carrying a duplication (Bainbridge \& Roper, 1966; Nga \& Roper, 1968), the translocation is probably reciprocal (Upshall \& Käfer, 1974). Further analyses are required to establish whether the translocation constitutes one of the ' + ' mutations in B6-27 or whether it was accidentally selected.

The relative yield improvements over their unselected ancestors shown by B6-27 and A6-9 in the present experiments (Table 4, Fig. 1) were lower than those obtained previously (Simpson \& Caten, 1979b). This could result from a change either in the fermentation conditions or in the strains, and no firm distinction between these two causes is possible from the information available. However, strain degeneration is considered unlikely in view of the homogeneity of the two strains (Table 2) and their storage on silica gel, a condition associated with little loss of viability (Perkins, 1962; Roberts, 1969). A more likely explanation is that some minor change in the fermentation conditions reduced the difference between the selected and unselected strains. Merrick (1975a) found that the difference in titre between natural high and low penicillin-producing isolates of $A$. nidulans was dependent on the batch of corn steep liquor used in the fermentation medium.

The type of control revealed in this analysis of strains selected by recurrent mutation 
and selection has several implications for the industrial application of hybridization and recombination techniques to raising the yields of antibiotics. Firstly, the non-allelism of the titre-increasing mutations in independently selected strains should stimulate attempts to obtain further improvement by hybridization of such strains. Since different genes are involved, the efficiency of the recombinational systems and the combining ability of the mutations are likely to be the two major factors determining the recovery of improved segregants from crosses. Hamlyn \& Ball (1979) have recently reported the isolation of industrially useful progeny from a cross between divergent strains of Cephalosporium acremonium, thereby demonstrating the practical value of hybridization. Secondly, the recessive or semi-dominant nature of titre-increasing mutations, together with the observed low productivity of the inter-strain diploid, reinforce the conclusion (Ball, 1978) that diploids between divergent lines are themselves unlikely to be useful and that improved strains should be sought amongst the haploid segregants from such diploids. Finally, the involvement of many genes means that the genetics of product yield in divergent crosses will be complex and will be analysed most efficiently by quantitative techniques (Caten \& Jinks, 1976; Caten, 1979). Genetic complexity is already apparent from the modest programmes used to select strains A6-9 and B6-27.

We thank Glaxo-Group Research Ltd, Sefton Park, Stoke Poges, Buckinghamshire for the provision of materials and the Glaxo staff for their guidance and encouragement. We are particularly grateful to Dr J. Croft for assistance with the tests for translocations and to Dr A. Upshall for supplying cultures. One of us (I.N.S.) gratefully acknowledges a Science Research Council CASE studentship.

\section{REFERENCES}

Alikhanian, S. I. (1962). Induced mutagenesis in the selection of microorganisms. Advances in Applied Microbiology 4, 1-50.

Alikhanian, S. I. \& Kameneva, S. V. (1961). Hybridisation of highly active strains of Penicillium chrysogenum. Scientific Reports of the Istituto Superiore di Sanità 1, 454-463.

BaCkus, M. P. \& STAufFer, J. F. (1955). The production and selection of a family of strains in Penicillium chrysogenum. Mycologia 47, 429-462.

Bainbridge, B. W. \& Roper, J. A. (1966). Observations on the effects of a chromosome duplication in Aspergillus nidulans. Journal of General Microbiology 42, 417-424.

Ball, C. (1971). Haploidization analysis in Penicillium chrysogenum. Journal of General Microbiology 66, 63-69.

BALL, C. (1973a). Improvement of penicillin productivity in Penicillium chrysogenum by recombination. In Genetics of Industrial Microorganisms, vol. II, pp. 227-237. Edited by Z. Vaněk, Z. Hoštálek \& J. Cudlín. Prague: Academia.

BaLl, C. $(1973 b)$. The genetics of Penicillium chrysogenum. Progress in Industrial Microbiology 12, 47-72.

BaLl, C. (1978). Genetics in the development of the penicillin process. In Antibiotics and Other Secondary Metabolites, pp. 165-176. Edited by R. Hütter, T. Leisinger, J. Núesch \& W. Wehrli. London: Academic Press.

BALL, C. \& AzEVEDo, J. L. (1976). Genetic instability in parasexual fungi. In Second International Symposium on the Genetics of Industrial Microorganisms, pp. 243-251. Edited by K. D. Macdonald. London: Academic Press.

Barratt, R. W., Johnson, G. B. \& Ogata, W. N. (1965). Wild-type and mutant stocks of Aspergillus nidulans. Genetics 52, 233-246.

CATEN, C. E. (1979). Quantitative genetic variation in fungi. In Qhantitative Genetic Variation, pp. 35-59. Edited by J. N. Thompson, Jr \& J. M. Thoday. New York: Academic Press.

CATEN, C. E. \& Jinks, J. L. (1976). Quantitative genetics. In Second International Symposium on the Genetics of Industrial Microorganisms, pp. 93-111. Edited by K. D. Macdonald. London: Academic Press.

Clutterbuck, A. J. (1973). Gene symbols in Aspergillus nidulans. Genetical Research 21, 291-296.

Clutterbuck, A. J. (1974). Aspergillus nidulans. In Handbook of Genetics, vol. 1, pp. 447-510. Edited by R. C. King. New York: Plenum Press. Demain, A. L. (1973). Mutation and the production of secondary metabolites. Advances in Applied Microbiology 16, 177-202.

Ditchburn, P., Holt, G. \& Macdonald, K. D. (1976). The genetic location of mutations increasing penicillin yield in Aspergillus nidulans. In Second International Symposium on the Genetics of Industrial Microorganisms, pp. 213-227. Edited by K. D. Macdonald. London: Academic Press. 
Elander, R. P. (1967). Enhanced penicillin biosynthesis in mutant and recombinant strains of Penicillium chrysogenum. Abhandlungen der Deutschen Akademie der Wissenschaften zu Berlin, Klasse für Medizin 2, 403-423.

Elander, R. P. \& Espenshade, M. A. (1976). The role of microbial genetics in industrial microbiology. In Industrial Microbiology, pp. 192-256. Edited by B. M. Miller \& W. Litsky. New York: McGraw-Hill.

Elander, R. P., Espenshade, M. A., Pathak, S. G. \& PAN, C. H. (1973). The use of parasexual genetics in an industrial strain selection programme with Penicillium chrysogenum. In Genetics of Industrial Microorganisms, vol. II, pp. 239-253. Edited by Z. Vaněk, Z. Hoštálek \& J. Cudlín. Prague: Academia.

Hamlyn, P. F. \& Ball, C. (1979). Recombination studies with Cephalosporium acremonium. In Genetics of Industrial Microorganisms, pp. 185-191. Edited by O. K. Sebek \& A. I. Laskin. Washington, D.C.: American Society for Microbiology.

Hastie, A. C. (1970). Benlate-induced instability of Aspergillus diploids. Nature, London 226, 771.

Holt, G. \& Macdonald, K. D. (1968a). Penicillin production and its mode of inheritance in Aspergillus nidulans. Antonie van Leewenhoek 34, $409-416$.

Holt, G. \& MaCdonald, K. D. (1968b). Isolation of strains with increased penicillin yield after hybridisation in Aspergillus nidulans. Nature, London 219, 636-637.

Holt, G., Edwards, G. F. St. L \& Macdonald, K. D. (1976). The genetics of mutants impaired in the biosynthesis of penicillin. In Second International Symposium on the Genetics of Industrial Microorganisms, pp. 199-211. Edited by K. D. Macdonald. London: Academic Press.

Hopwood, D. A. \& Merrick, M. J. (1977). Genetics of antibiotic production. Bacteriological Reviews 41, 595-635.

KÄFER, E. (1962). Translocations in stock strains of Aspergillus nidulans. Genetica 33, 59-68.

LHOAS, P. (1961). Mitotic haploidisation by treatment of Aspergillus niger diploids with p-fluorophenylalanine. Nature, London 190, 744.

MaCDONALD, K.D. (1968). The persistence of parental genome segregation in Penicillium chrysogenum after nitrogen mustard treatment. Mutation Research 5, 302-305.

Macdonald, K. D. \& Holt, G. (1976). Genetics of biosynthesis and overproduction of penicillin. Science Progress 63, 547-573.

Macdonald, K. D., Hutchinson, J. M. \& Gillett, W. A. (1963a). Heterokaryon studies and the genetic control of penicillin and chrysogenin production in Penicillium chrysogenum. Journal of General Microbiology 33, 375-383.

Macdonald, K. D., Hutchinson, J. M. \& Gillett, W. A. $(1963 b)$. Formation and segregation of heterozygous diploids between a wild-type strain and derivatives of high penicillin yield in Penicillium chrysogenum. Journal of General Microbiology 33, 385-394.

Macdonald, K. D., Hutchinson, J. M. \& GLletT, W. A. (1964). Properties of heterozygous diploids between strains of Penicillium chrysogenum selected for high penicillin yield. Antonie van Leeuwenhoek 30, 209-224.

Macdonald, K.D., Hutchinson, J. M. \& Gillett, W. A. (1965). Heterozygous diploids of Penicillium chrysogenum and their segregation patterns. Genetica 36, 378-397.

MCCully, K. S. \& Forbes, E. (1965). The use of $p$-fluorophenylalanine with "Master strains" of Aspergillus nidulans for assigning genes to linkage groups. Genetical Research 6, 352-359.

MERRICK, M. J. (1975a). Hybridization and selection for increased penicillin titre in wild-type isolates of Aspergillus nidulans. Journal of General Microbiology 91, 278-286.

MerRICK, M. J. (1975 b). The inheritance of penicillin titre in crosses between lines of Aspergillus nidulans selected for increased productivity. Journal of General Microbiology 91, 287-294.

Merrick, M. J. \& Caten, C. E. (1975). The inheritance of penicillin titre in wild-type isolates of Aspergillus nidulans. Journal of Genral Microbiology 86, 283-293.

MuCHNIK, R. A. (1961). Induced somatic segregation and chromosome mapping in Penicillium chrysogenum. Aspergillus Newsletter 2, 7.

NGA, B. H. \& Roper, J. A. (1968). Quantitative intrachromosomal changes arising at mitosis in Aspergillus nidulans. Genetics 58, 193-209.

Perkins, D. D. (1962). Preservation of Neurospora stock cultures with anhydrous silica-gel. Canadian Journal of Microbiology 8, 591-594.

Pontecorvo, G. \& Sermonti, G. (1954). Parasexual recombination in Penicillium chrysogenum. Journal of General Microbiology 11, 94-104.

Pontecorvo, G., Roper, J. A., Hemmons, L. M., Macdonald, K. D. \& Bufton, A. W. J. (1953). The genetics of Aspergillus nidulans. Advances in Genetics 5, 141-253.

RoBerts, C. (1969). Silica gel stock cultures of Aspergillus nidulans. Aspergillus Newsletter 10, 29.

ROPER, J. A. (1952). Production of heterozygous diploids in filamentous fungi. Experientia 8, 14-15.

RoPER, J. A. (1973). Mitotic recombination and mitotic non-conformity in fungi. In Genetics of Industrial Microorganisms, vol. II, pp. 81-88. Edited by Z. Vaněk, Z. Hoštálek \& J. Cudlín. Prague: Academia.

Sermonti, G. (1957). Analysis of vegetative segregation and recombination in Penicillium chrysogenum. Genetics 42, 433-443.'

Sermont, G. (1959). Genetics of penicillin production. Annals of the New York Academy of Sciences 81, 950-966.

SERMONTI, G. (1961). The parasexual cycle in Penicillium chrysogenum and its application to the production of penicillin. Scientific Reports of the Istituto Superiore di Sanità 1, 449-454.

Sermont,, G. (1969). Genetics of Antibioticproducing Microorganisms. London: Wiley Interscience.

Simpson, I. N. \& Caten, C. E. (1979a). Induced quantitative variation for penicillin titre in clonal populations of Aspergillus nidulans. Journal of General Microbiology 110, 1-12.

Simpson, I. N. \& CATEN, C. E. (1979b). Recurrent mutation and selection for increased penicillin 
titre in Aspergillus nidulans. Journal of General Microbiology 113, 209-217.

Thoday, J. M. \& Thompson, J. N., Jr (1976). The number of segregating genes implied by continuous variation. Genetica 46, 335-344.
Upshall, A. \& KÄFER, E. (1974). Detection and identification of translocations by increased specific non-disjunction in Aspergillus nidulans. Genetics 76, 19-31. 